\title{
Sensor Information Management using Cloud Computing
}

\author{
K. Surya Bharat \\ Karunya University \\ Coimbatore,INDIA
}

\author{
A.Naga Priyanka, V.R.Siddhartha \\ Vijayawada,INDIA
}

\begin{abstract}
Wireless Sensor Networks (WSN) and cloud computing are the present day technologies which enable novel and attractive solutions for information gathering and accessing it across the globe. There is a meticulous research going on in the labs and universities on these areas. However there are obstacles that must be overcome before the full potential of this can be realized. One such obstacle is the sensor data cannot be accessed globally. The insight of this paper is to elaborate and analyze the critical issues in WSN and which can be solved by integrating it with the cloud. The main objective of this paper is to make sensor data available across the globe and reduce the maintenance costs.
\end{abstract}

\section{Keywords}

Cloud computing, Wireless Sensor Networks, Information management using cloud, Sensor management, Software as a Service (SaaS), Platform as a Service (PaaS), Infrastructure as a Service (IaaS).

\section{INTRODUCTION}

The term "Cloud" has evolved from illustrations which depicted remote environment as cloud images, in order to conceal the complexity that lies behind them. The Cloud Computing is an all-embracing and rapidly evolving concept. A plethora of definitions are given to explain Cloud Computing. Some of them are discussed below.

Cloud Computing is defined as "A technology for enabling convenient, on demand network access to a shared pool of computing resources" [5].In Cloud Computing, the processing is done at the server level and not at the desktop level. Desktop is a conduit terminal. Cloud Computing is dynamic, so it helps companies to enlarge or dwindle the computational resources quickly and efficiently without much loss of money spend on infrastructure [13].

Cloud computing mostly relies on virtualization and grid computing. Virtualization has been an underlying concept towards the development of the Cloud. Virtualization helps to render all the services, supported by the hardware that can be observed on a personal computer, to the end user [5]. Cloud computing helps users to access information and computational resources from any part of the globe if there does exist a reliable internet connection. Cloud Computing provides a shared pool of resources, including data storage space ,networks, computer processing power and specialized corporate and user applications.

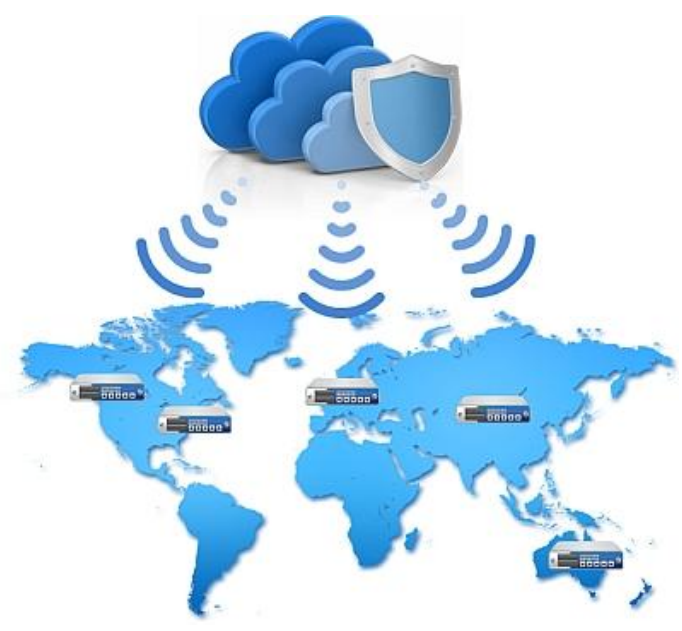

Fig 1: Depiction of Cloud Computing

There is an instant shift to Cloud Computing from all the sectors. By adopting Cloud Computing it will be a benefit to many small and medium industries where establishment of IT infrastructure is a hassle. Cloud Computing is "pay-as-yougo" type, so the organization have the flexibility to expand or decrease the amount of infrastructure quickly and efficiently.

\section{INDUSTRY PROSPECTIVE OF CLOUD}

There are expeditious transforms in the contemporary business market around the globe .The need to respond for the business requisites are imperative in the present scenario. Waiting for establishment of new servers is unacceptable and it may even cause us to lose our customers. Predominantly Cloud Computing helps all Small and Medium scale Enterprises where budget is a main concern. Small and medium scale enterprises play a vital role in generating employment opportunities [11]

Cloud Computing can significantly reduce the cost and complexity of owning and operating computer and networks. In addition Cloud providers that have specialization in a specific area can bring advancement in services that a single company might not be able to afford. Moreover by using cloud the companies need not worry about mundane concerns like Software up-gradation and other issues.

Cloud Computing, virtualization and grid computing are connected to each other. Wireless Sensor Networks integrating with the Grid computing is not appropriate for most purposes. For an instance, grid computing focuses on high performance, while cloud concentrates on general purpose application [13]. 
This generic nature of cloud makes it more attractive and perfectly suitable for integrating it with the WSN.

There are many Cloud providers in the present day market, prominent among them are Google (Google apps), Amazon (Simple storage service-S3), IBM, Microsoft etc [8].

Cloud computing not only benefits the organization but also proves its usefulness to the environment. By adapting to Cloud, there is decrease in consumption of hardware which in turn will be a reduction of waste and eventually reduce the emission of carbon in our environment [15].

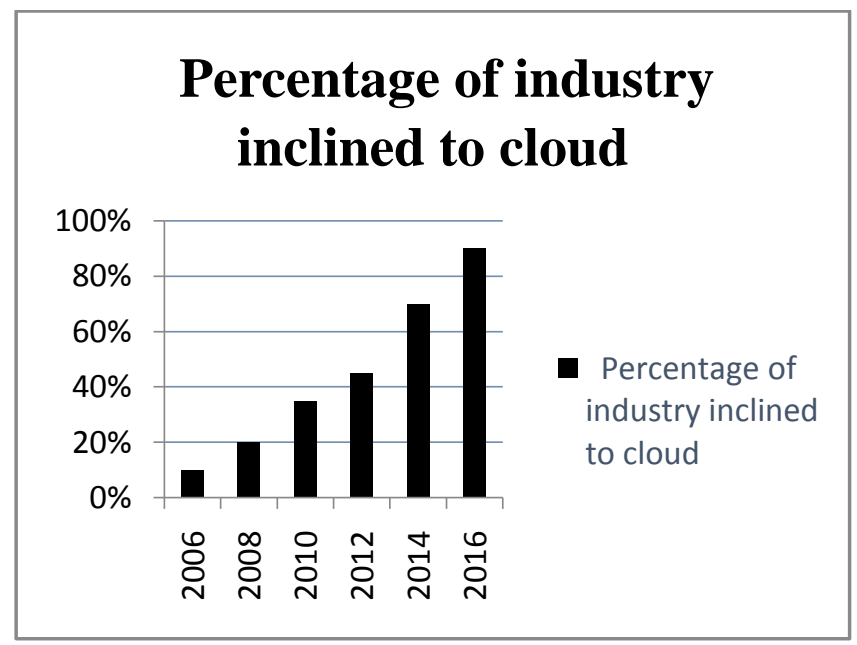

Fig 2: Part of industry shifting to cloud

\section{CLOUD CLASSIFICATION}

Cloud Computing can be classified based on the services offered and deployment models.

According to the services offered, Cloud Computing consists of three layers. Infrastructure as a Service (IaaS) is the lowest layer that provides basic infrastructure support service. Platform as a Service (PaaS) layer is the middle layer, which offers platform oriented services, besides providing the environment for hosting user's applications. Software as a Service (SaaS) is the topmost layer which features a complete application offered as service on demand [1]. Many products that are offered by these three layers require some degree of programming in order to access the functionalities that exists in these services [8].

The deployment models of the cloud depend on the customer's requirement. In Public Cloud the infrastructure is provided to many customers and is managed by the third party. In Private Cloud infrastructure is provided to only limited customers and in many cases it is managed by the Organization itself. In Community Cloud the resources are shared by several organizations or a group of people. Now comes the present day highly used type of cloud, it is Hybrid Cloud [11]. It is a composition of two or more cloud deployment models. So it has better scope than the other existing types.

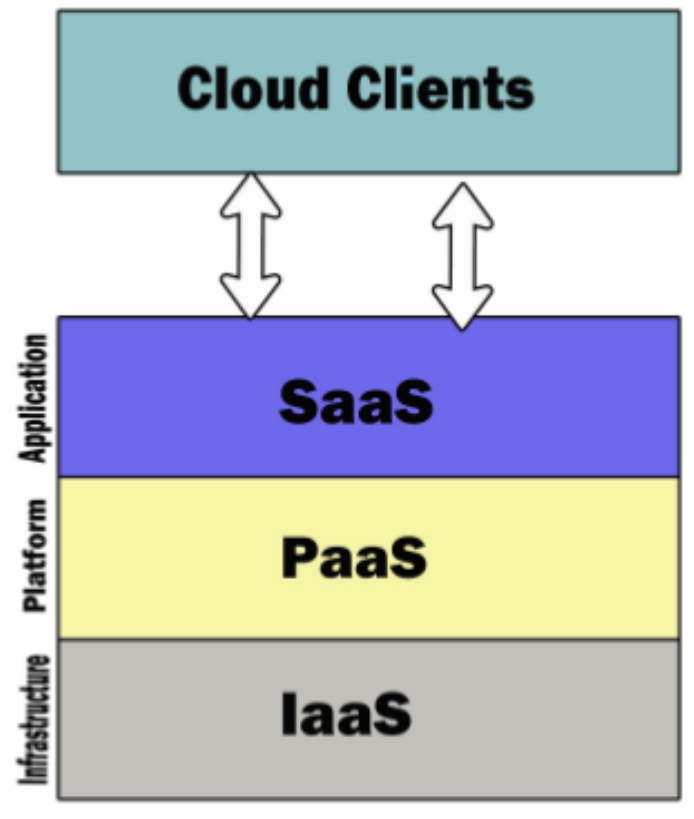

Fig 3: Services offered in Cloud

\section{SENSOR INFORMATION MANAGEMENT}

Sensor networks are conceded as one of the most paramount technologies that has come so far. In the present day environment Sensor networks are used in myriad applications. For an instance they are used for environment monitoring, manufacturing industries, security checks and many more. Currently we implement Virtualization concept in sensors. Sensor Virtualization technology enables one physical sensor to be projected as multiple virtual sensors [9]. It also helps users to employ sensor data in a transparent manner.

Data that emanate from these sensors should be aggregated, managed, stored, and mined in the right way so that we can obtain optimum benefit from it. The data obtained from these sources is "stream data" i.e. much of the data is transient[12].So SIM system has to rapidly analyze and discard the data that is not needed and stores the essential ones.

Sensor Information Management is a component of the Sensor network. There is a subtle difference between Sensor Data Management and Sensor Information Management [9]. Sensor data management is to manage, analyze and process data stream form the sensors, and sensor information management is to organize, analyze, mine and apply sensor data. Typically Sensor Information Systems encompasses both SIM and Sensor Data Management systems [12].

We need distributed data processing capabilities for managing distributed Sensors .Each sensor may have its own data management system and various data management systems may be connected to share the data. In some cases the data will be aggregated from all the sensors and sent to the central data management system for processing. Aggregating the data emanating from these sensors and making sense out of it is a hectic task. At times data may be incomplete or inaccurate. So the Sensor Management System should be disposed to deal with the incomplete data. 
Typically there are two types of methods employed for emanating the data from sensors; they are static and dynamic method [37]. In static method the data will be collected and stored for future use. Here the data need not to be processed immediately but the data is stored in the data base for future reference. In dynamic method the data emanating should be processed within a certain time limit, if not there might be a great loss. For instance the data obtained from the surveillance cameras at airports and other places should be checked instantly within certain time limit.

Cloud computing services render users with virtual servers. Users utilize virtual servers without being concerned about their location and specifications. Moreover users can also control sensors through virtual servers, irrespective of their applications.

Usually sensors are deployed in a monitored space and send their sensor data to local database. But if we employ this innovative model we can send data directly to Cloud through cloud gateways which can be used from any part of the world provided with a reliable internet connection

\subsection{Wireless Sensor Network (WSN) and its Applications}

In the present day scenario Wireless Sensor Networks are used in myriad of devices for making the work of the individuals easier. For an instance they are used in security and surveillance, environment monitoring, health applications and many more.

Cloud Computing is primarily designed and promoted to be data centric and efficient interaction with the outside world. Wireless Sensor Networks are used to collect data in the real world, but the data which is obtained from the WSN is massive after piling up for some time. This organization may need the data for future use but right now it is of redundant for them. So they should store this data safely so that they can retrieve whenever it is required.

Data collectors (loggers) are the devices which gather information from the environment. Traditional data loggers require regular manual supervision and recurrent site visits to collect data from them. But if we integrate WSN with the cloud, the sensors in the particular area sends the data continuously into the network through the cloud gate way and there is no manual interference is required and can be done automatically. This implementation requires minimum maintenance when in operation.

\subsection{Generic Sensor Node Architecture}

Typically, a sensor node is a tiny device that includes five main components in it [23][39].

1. A sensing subsystem for data acquisition from the surrounding environment

2. A processing subsystem for local data processing

3. A wireless communication subsystem for data transmission

4. A battery which supplies all the energy required for functioning

5. A memory for storing the collected data.

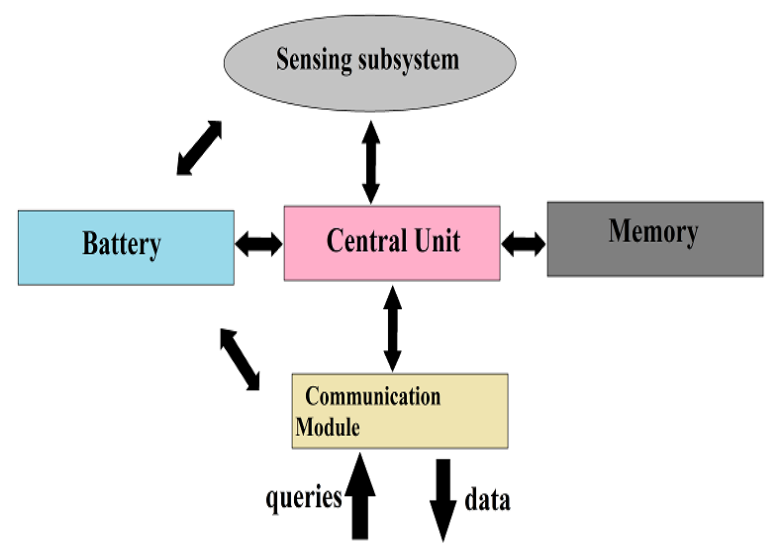

Fig 4: Architecture of Sensor node

In the present day environment there are two types of methods followed for analyzing and storing data in the wireless sensor networks.

In the first method, the sensor gathers all the information from the physical environment in which they are placed and then sends it to the local analyzing centre known as "data loggers", these are not just meant for collecting the information from the sensor but also they manage, analyze, and process the data. The users who are permitted to access this data are connected to the data loggers and access the data required by them.

By implementing this method there are a couple of drawbacks which lead us to think innovatively how to overcome them in our model. The main drawback is that the distance between the sensor and data logger should not exceed the maximum length, if this happens the data logger cannot receive the information that is sent by the sensors [36]. This information is lost and cannot be retrieved. This is not only a loss of important data but also a waste of energy of the sensor in transmitting the information. In crucial systems even a loss of data from one sensor is also considered important. The failure of one node may interrupt the entire system or application.

The second limitation is that this implementation is not global i.e. the people who are just around the WSN system could only get the info. This info cannot be accessed by all the people around the globe. Moreover the storage space in the data logger is fixed and scaling cannot be done. So after a certain time the old data should be erased for providing space for new data. This process is known as aging. Because of this process there is a loss of data which is old and might be useful in future.

In the second method, the information collected by sensor will not be sent to the data logger, instead the sensor itself will analyze, manage and process the data into necessary output. 


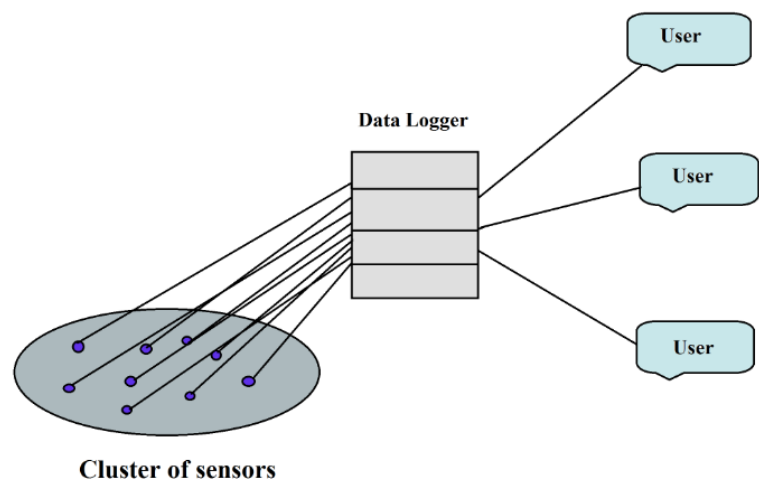

\section{Fig 5: Information management using first conventional} method

Here all the computational work is done by the sensor itself. It only sends the output to the data logger through which the user interacts and collects the required information. But by implementing this method there are some issues which leads to decrease in performance of sensors.

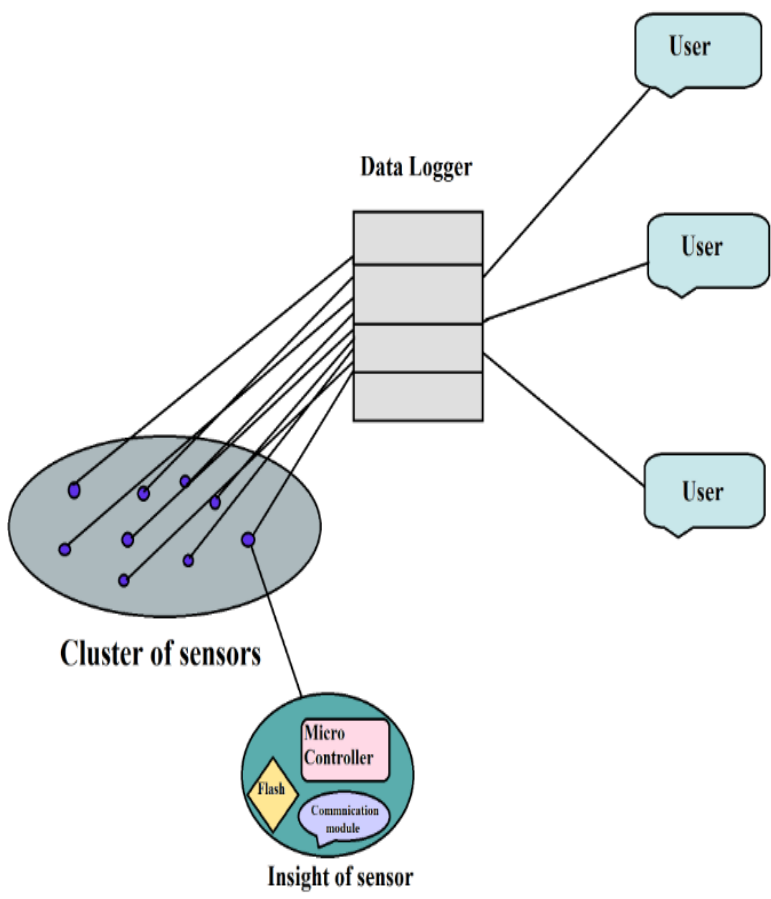

Fig 6: Information management using embedded sensor method

The main idea of this paper is to propose an ingenious method of managing the sensor data without regular discharge of battery. This problem arises due to not letting the information send to the data loggers for computation, rather it is being done by sensor itself. Because of this extra work load on the sensor its battery discharges quickly, which will eventually make the sensor futile. In distributed sensor systems the battery life of the sensor should be more so that there is less maintenance for it. Moreover if one sensor becomes defunct, this might affect the output, as each and every sensor is equally important in ideal functioning of application.
The second issue in implementing the above method is the use of flash memory in the sensors [37]. The flash memory used here is very limited, so that the sensor cannot store all the data of the past. As the flash starts filling up, some data needs to be discarded to make room for the upcoming data. Flash memories are considered to be less energy efficient than the conventional ones. These are different from magnetic disks in architecture, energy constraint etc. The main drawback in flash memory is once the data is written; a memory location must be reset or erased to use it again.

\section{PROPOSED ARCHITECTURE}

The objective of this paper is to eventually shift the data of WSN to cloud Environment so that the valuable data can be optimally used at any point of time from anywhere.

In our proposed model unlike the above two models the Wireless Sensor Network, eventually shifts the data into the cloud and overtime into the public domain. By implementing this we can get information of the sensors from any part of the world if we are connected to internet. Users will connect to cloud through a secured Identity and Access Management Unit (IAMU) and will be given access on the basis of the policy framed against their user accounts. IAMU will provide both authentication and access control to the users.

WSN are easy to install, flexible to move from one position to another and easy to manage.WSN continuously collect a wide range of information required by the users . The collected information is passed over a network and stored in the Cloud and made available via Internet for instant access.

There is an intense ongoing research in this field of study .In this paper we scrutinized the previous methods thoroughly and proposed a innovative technique which can overcome the pitfalls of the conventional architectures.

In this method the sensors don't analyze the data by themselves or send it to the local data logger for computation, instead they send the data directly to cloud through cloud gateways. The gateway is like a sink or temporary buffer, through which the data is passed on to the cloud. By implementing this method the battery life of the sensor can be improved drastically. All the computational work is done in the cloud. So the organization need not worry about the infrastructure. The cloud storage is elastic i.e. it can be enlarged or dwindled depending on the necessity of the organization. This saves the investment on infrastructure. In this process the memory is unlimited which makes the sensor to store all the information in cloud without any loss.

By implementing this method, the data that is gathered by the sensors can be accessed from any part of the globe, by having a reliable internet connection through the cloud database. The access eligibility is given by the cloud providers depending on the agreements they make with the users. There is no limitation of the distance between the sensor and the data logger if this method is employed. The user can control and supervise all the activities at the click of the mouse. 


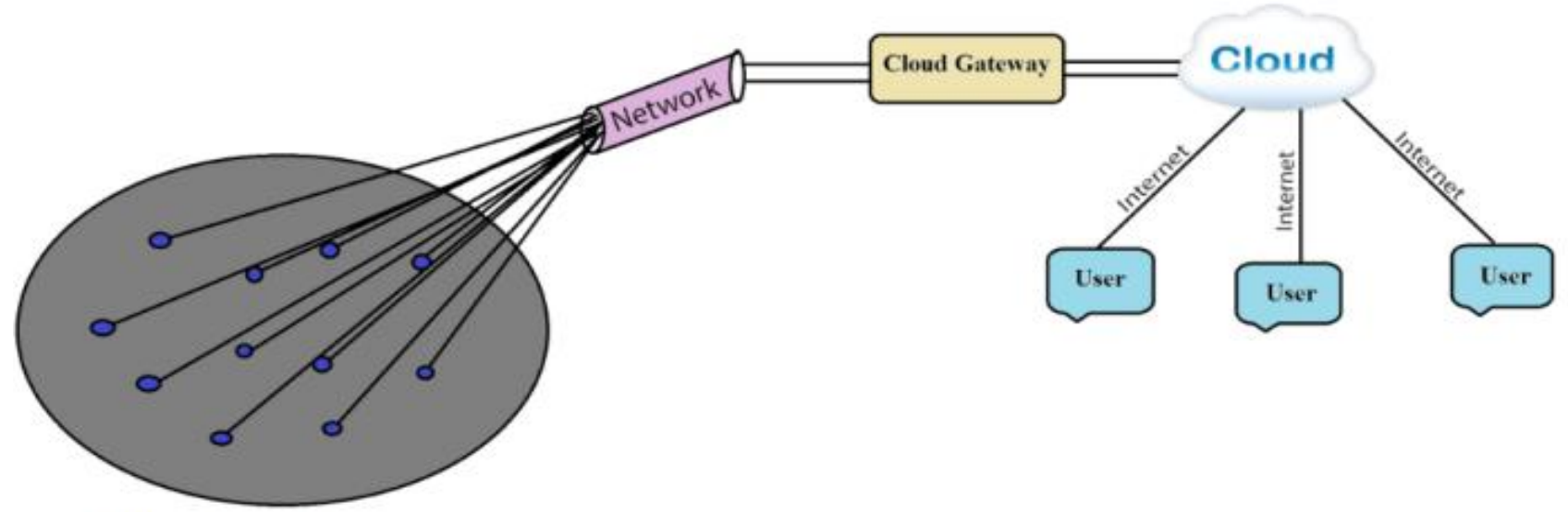

Cluster of sensors

Fig 7: Proposed architecture of integrating WSN with Cloud computing.

The cloud can be accessed by the authorized candidates only, so the information is highly secure. The data reaches the cloud through cloud gate ways. As the cloud have huge amount of data storage, all the history of the data can be stored for future purposes.
Some of the data which reaches the cloud should be analyzed and sent to the users immediately. Here the data should reach the user in time. For an instance the surveillance data in airports, malls etc.

Table 1: Comparative analysis of conventional method with the proposed architecture

\begin{tabular}{|c|c|c|c|}
\hline Attribute & Conventional method & $\begin{array}{c}\text { Embedded Sensor } \\
\text { method }\end{array}$ & Cloud Gateway method \\
\hline Cost of Infrastructure & High & High & Very less \\
\hline Memory Storage & Moderate & Very Less & High \\
\hline Battery Life & Average & Very Less & Less required \\
\hline Maintenance & Frequent & Frequent & Across the globe \\
\hline Access of Data & Limited by distance & Limited by distance & Readily available \\
\hline Flexibility & Hard and costly & Hard and costly & Very much high \\
\hline Process Speed & Moderate & Depends on Hardware & \\
\hline
\end{tabular}

\section{FUTURE SCOPE AND CHALLENGES IN CLOUD AND WSN}

Every invention has its pros and cons, in the similar way, although there are many advantages with cloud computing technology there are some minor drawbacks which can be overcome if dealt scrupulously [23]. Some of the barriers are

1. Loss of control of data

2. Legal issues

3. Possible downtime without internet connection

4. Security and privacy issues.
The main issue is that the managers of the organization feel wariness about the confidential data as it is stored in the third person's hand. They are also worried about the server break down which is responsible for loss of service to customers.

There are some legal issues like suspension of end users accounts ,un authorized or inappropriate use etc that has to be addressed to get optimum results from cloud computing. There are times when the servers crashed and the transfer of data is stopped for certain amount of time. So precautions should be taken to overcome these issues effectively by keeping the duplicate data in other servers which can be kept as backup.

The cloud providers should maintain the standards and proper security firewalls, so that no unauthorized person can access the data of the customers. They can also make the customers to access the cloud storage by providing their credentials whenever they wish to use it. 
But all of these drawbacks can be overcome by implementing the following in cloud network: increasing the level of security in the cloud, examining the cloud provider security policy closely and initiating organizations like The Cloud Security Alliance to collaborate with cloud experts and increase the security assurance in cloud computing.

There are also other concerns like random topology and hostile environment for WSN which can be overcome if dealt in a proper procedure. Sometimes the sensor networks will be deployed in war fields. In these cases, the nodes cannot be protected from physical attacks. So care should be taken for proper replacement of the nodes immediately for getting the data without any losses. Sometimes sensors are deployed in random topology from an airplane. In these situations, it is hard to store various encryption keys on nodes in order to establish encryption among a group of neighbours, since the neighbourhood cannot be known priories. Proper care should be taken to design key agreement protocols that do not require certain nodes to be neighbours of some other nodes, and also do not require encryption keys to be stored on sensors before deployment.

\section{CONCLUSION}

The cloud computing and sensor networks are dominating the market in the present day scenario. Many analysts have found that there will be a major shift towards cloud in the coming years. The advantage of cloud over the grid and cluster computing is that it is generic. So this can be easily integrated with Wireless Sensor Networks. In the present day WSN are used without the integration of cloud. So their data access across the world has become a main issue. So we came up with the idea of integrating the WSN with cloud and make the data process and store in cloud, so that there is low latency, flexibility and data can be accessed around the world.

Although Cloud Computing and WSN has revolutionized the world, there are some security issues in cloud and environmental issues in WSN which should be addressed scrupulously. These can be overcome by increasing the security in the cloud and replacing the damaged nodes in the hostile environments for WSN. By implementing all these security measures, we can make cloud computing ideal for all the business across the globe.

\section{ACKNOWLEDGEMENTS}

The authors are extremely thankful to Mr. Venkateshan, Mrs. Rama Lakshmi and Mr. Mathew Kurian for their invaluable support and encouragement throughout the course of this paper. At last but not the least we want to thank our parents who treasured us for our hard work and encouraged us and finally to God who made all things possible for us till the very end.

\section{REFERENCES}

[1] Manas M, Nagalakshmi C, Shobha April 2014 "Cloud Computing Security Issues And Methods to Overcome".

[2] Ezer Osei Yeboah-Boateng, Kofi Asare Essandoh February 2014 "Factors Influencing the Adoption of Cloud Computing by Small and Medium Enterprises in Developing Economies".

[3] L.Mihaylova,A.Nix,D.Angelova,D.Bull,A.Munro, Canagarajah "Information Management Methods in Sensor Networks".
[4] Brendan Jennings and Rolf Stadler "Resource Management in Clouds: Survey and Research Challenges".

[5] Rohit Bhadauria, Sugata Sanyal "Survey on Security Issues in Cloud Computing and Associated Mitigation Techniques".

[6] Khandakar Entenam Unayes Ahmed, Mark A Gregory "Integrating Wireless Sensor Networks with Cloud Computing".

[7] Jaeseok Shim and Yujin Lim1 June 2013 " Implementation of Real Time Alert System over Cloud computing".

[8] Nabil Sultan 2009"Cloud computing for education: A new dawn?"

[9] Pengfei You, Yuxing Peng "An Architecture Model of Sensor Information System Based on Cloud Computing”.

[10] Laura DuBois, Richard L. Villars, and Brad Nisbet June 2011 "Optimizing Information Management in the Cloud ".

[11] Reza Sahandi, Adel Alkhalil, Justice Opara "Cloud computing from SMES prospective a survey based investigation".

[12] Bhavani Thuraisingham "An overview of infrastructures and data managers for dependable sensor networks".

[13] Khandakar Entenam Unayes Ahmed, Mark A Gregory "Integrating Wireless Sensor Networks with Cloud Computing"

[14] Wen-Yaw Chung, Pei-Shan Yu, Chao-Jen Huang 2013 "Cloud Computing System Based on Wireless Sensor Network".

[15] Cong Wang, Qian Wang, and Kui Ren "Ensuring Data Storage Security in Cloud Computing".

[16] QiHana,, Sharad Mehrotrab, Nalini Venkatasubramanian May 2007 "Application-aware integration of data collection and power management in wireless sensor networks".

[17] Yanlei Diao, Deepak Ganesan, Gaurav Mathur, and Prashant Shenoy "Rethinking Data Management for Storagecentric Sensor Networks".

[18] T. Ravi Kumar and K. Raghava Rao March 2013 "Sensor data analysis and management in wireless sensor networks".

[19] David Chu,Amol Deshpande§ Joseph M. Hellerstein,Wei Hong "Approximate Data Collection in Sensor Networks using Probabilistic Models".

[20] Ousmane Diallo, JoelJ.P.C.Rodrigues, MbayeSene December 2011 "Real time data management on wireless sensor network : A survey"

[21] Vongsagon Boonsawat, Jurarat Ekchamanonta, Kulwadee Bumrungkhet, and Somsak Kittipiyakul "XBee Wireless Sensor Networks for Temperature Monitoring".

[22] Quan Le-Trung, Dr.techn October 2012 "Research areas in Wireless Sensor Networks".

[23] Madhav Bokare, Anagha Ralegoonkar January 2012 " Wireless Sensor Network: A Promising Approach for Distributed Sensing Tasks." 
[24] Quan Le-Trung, Dr.techn "Research Areas in Wireless Sensor Networks".

[25] OmidBushehrian,YousefEmami, Reza Javidan"Automatic Management of Wireless Sensor Networks through Cloud Computing".

[26] B.Thirumala Rao, Dr. L.S.S.Reddy “ Survey on Improved Scheduling in Hadoop MapReduce in Cloud Environments".

[27] Vijijdra, Sudhir senai "Survey On Scheduling Issues in Cloud computing".

[28] Nelson Gonzalez, Charles, Fernando Red'igolo, Marcos Simpl, Tereza Carvalho, Mats N"aslund2 and Makan Pourzandi 2012 "A quantitative analysis of current security Concerns and solutions for cloud computing".

[29] Mohamed Magdy Mosbah1, Hany Soliman , Mohamad Abou El-Nasr " Current Services In Cloud Computing : A Survey".

[30] Jingwei Huang and David M Nicol "Trust mechanisms for cloud computing".

[31] Dana Petcu1, Beniamino Di Martino, Salvatore Venticinque2, Massimiliano Rak2, Tamas Mahr,Gorka Esnal Lopez, Fabrice Brito, Roberto Cossu, Miha Stopar, Svatopluk Šperka and Vlado Stankovski "Experiences in building a mosiac of clouds".
[32] Rizwana Shaikh, M. Sasikumar "Security Issues in Cloud Computing: A survey".

[33] Misastephan "Cloud Computing: What It Is and Where to Start".

[34] Yi Zou and Krishnendu Chakrabarty 2003 "Sensor Deployment and Target Localization Based on Virtual Forces".

[35] Wen-Yaw Chung, Pei-Shan Yu, Chao-Jen Huang "Cloud Computing System Based on Wireless Sensor Network".

[36] Yanlei Diao, Deepak Ganesan, Gaurav Mathur, and Prashant Shenoy "Rethinking Data Management for Storagecentric Sensor Networks"

[37] Ousmane Diallo, JoelJ.P.C.Rodrigues , MbayeSene "Real-time data management on wireless sensor network: A survey".

[38] Amal H. Al-Gamdi1 \& Ibrahiem M. M. El Emary “ The Vital Role of Wireless Sensors Networks in Potential Applications".

[39] Ant'onio J. Pires, Erico Meneses Lẽao, Jo ao P. Sousa, Luiz Affonso Guedes, and Francisco Vasques "RealTime Communication for Smart Sensor Networks: A CAN Based Solution". 